\title{
SATURATION OF ALFVEN OSCILLATIONS IN THE RING CURRENT REGION DUE TO GENERATION OF LOWER HYBRID WAVES
}

\author{
K. V. GAMAYUNOV, E. N. KRIVORUTSKY and A. A. VERYAEV \\ Altai State University, 66 Dimitrov St, 656099 Barnaul, Russia \\ and \\ G. V. KHAZANOV \\ Space Physics Research Laboratory, University of Michigan, 2455 Hayward, \\ Ann Arbor, MI 48109, U.S.A.
}

(Received in final form 3 October 1991)

\begin{abstract}
The possibility of flux generation of lower hybrid oscillations in the ring current region of the Earth's magnetosphere is suggested in this paper. The energy level of lower hybrid oscillations can exceed the modulational instability threshold, which leads to the formation of caverns. The consequences of this are qualitatively analysed. Also, an assumption is made that the flux instability of lower hybrid oscillations may limit the level of Alfvén oscillations in the ring current region.
\end{abstract}

\section{INTRODUCTION}

It is common knowledge that the ring current has a significant effect on the dynamics of ionospheremagnetosphere interactions by generating MHD waves (Galeev, 1975; Kennel and Petschek, 1966; Lyons and Williams, 1984). In this connection, while describing interactions of waves with plasma particles. there arises the need to know the spectral energy density of Alfvén and fast magnetosonic waves (FMS). The concrete mechanisms of the formation of the spectra of MHD oscillations in the ring current zone (Young et al., 1981) are not yet clear. Usually, stabilization of oscillations is explained either by quasilinear interaction or by non-linear frequency shift (see Makarenko and Tupchenko, 1988, and references therein). The present paper examines the generation of lower hybrid oscillations (LHO) by Alfvén and/or FMS waves. The main consequence of this process is, in our view, the possibility of saturation of the energy level of MHD waves in the ring current zone.

Below we shall discuss the generation of LHO by Alfvén waves, since the mechanism of excitation of LHO is not dependent on the type of MHD oscillations (Akhiezer, 1974). Besides, the assertion about the saturation of the energy level of Alfven waves is equally true for FMS waves. This is connected with the fact that the presence of FMS waves in the ring current zone is explained by the linear transformation of Alfvén oscillations in a plasma with a small share of helium ions (Korth et al., 1984).
Experimental data (Taylor et al., 1975; Young et al., 1981) reveal that the energy density of Alfven waves fluctuates within the bounds of $10^{-5} \leqslant B^{2} / B_{0}^{2} \leqslant 10^{-4},(B$ is the magnetic field of the wave, $B_{0}$ is that of the Earth). As will be shown later, they can generate sufficiently intensive LHO. The study of this process, together with the possibility of saturation of the energy level of Alfvén oscillations, is of interest in understanding a number of magnetospheric phenomena.

First of all, note that LHO have apparently been observed on Dynamics Explorer I near the magnetic equator (Olsen et al., 1987). Evaluations that could be made on the basis of the results given in the paper, show a fairly high level of energy density of oscillations $W / n T \sim 10^{-6}-10^{-7}$ ( $W$ is the energy density of LHO; $n$ and $T$ are the concentration and temperature of the plasma). As is known, at such energy densities, LHO are unstable with respect to density modulation, which leads to strong turbulence and the collapse of individual caverns (Sturman, 1976). This means that excitation of LHO may lead to the appearance of an additional channel of energy transfer from Alfvén waves to particles, may influence the formation of the plasma particle distribution function at the expense of particle acceleration at the "tail" of the distribution function during the collapse, and influence the transport processes, since there occur additional collisions of particles with caverns and particle distribution functions which are changing (Musher et al., 1986). 


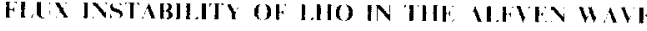 FIEI.1)}

As is known (Akhiczer. 1974) in the field of at lon frequency wave the drilt of electrons and ions oceurs with different velocity. II the relative velocity of electron and ion plasma components is high enough. $|\mathbf{u}|=\left|\mathbf{u}_{\mathrm{i}}-\mathbf{u}_{\mathrm{c}}\right|>r_{\mathrm{l}}$ then there occurs the flux instatbility of LHO. Here $r_{1 i}$ is the ion thermal speed. The instability is aperiodic

$$
R(1)-I m(1) \equiv \text { in }-V\left|(1)_{\mid k c}\right|()_{13 i} \equiv()_{1+1-}
$$

where (") ${ }_{\mathrm{BC}}$ and (") ${ }_{3 \mathrm{i}}$ are gyrofrequencies of electrons and ions, respectively. Numerical calculations along the trajectory of the Alfven wave propagation show that the condition $|\mathbf{u}|>r_{1 i}$ is carried out in a number of cases for the values of $B^{2} B^{2}$ observed in experiments in the ring current region (Taylor et al., 1975; Young (t al., 1981). Let us estimate the energy density of LHO. Generation takes place in the region of angles $\cos ^{2} \theta-m_{\mathrm{s}} m_{\mathrm{i}}(\theta)$ is the angle between the wave vector of $\mathrm{LHO} k$ and the magnetic field). At angles of $\cos ^{2} \theta \leqslant m_{\mathrm{c}} m_{\mathrm{i}}$ the main non-linear process, within the framework of the weak turbulence theory, is induced scattering of LHO on electrons with characteristic time $\left(\begin{array}{c}n \\ i \\ i\end{array}\right){ }^{\prime}$ (Musher et al., 1978).

The energy density of LHO, $W_{h}$ can be estimated from :

$$
\begin{aligned}
& \frac{\mathrm{d} W_{\mathrm{k}}}{\mathrm{d} t}=\left(\gamma_{\mathrm{n}}-\gamma_{\mathrm{d}}-\gamma_{\mathrm{i}}\right) W_{\mathrm{h}},
\end{aligned}
$$

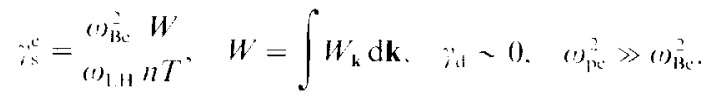

From the stationary condition we have

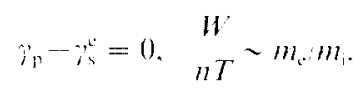

At these energy densities the plasma should not be considered weakly turbulent. since already at

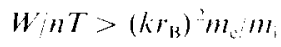

there occurs a modulational instability of LHO $\left(r_{\mathrm{B}}=r_{\mathrm{T}} / \omega_{\mathrm{BC}} . k\right.$ is the characteristic value of the wavenumber from the LHO spectrum) (Musher and Sturman, 1975). Since at such growth rates there is no point in differentiating between low frequency and high frequency oscillations, and the dynamics of this process have not yet been studied, ket us confine ourselves to the evaluation of the energy flux into the plasma (Musher el al., 1986).

$$
\underline{Q}=\omega_{1.11} H^{\prime}
$$

Hence:

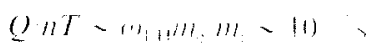

Thus, on separate sections of the Alfen wate rajetory, the above effect may lead to a significant heating of particles and possibly particle acecteration from the "latils" of the distribution function. The Jatler assumption needs additional analysis

For the effective frepuency of eollisions of plasma patrticks with caverns (o) $=v_{010} 47$ ). We have from cquation (5):

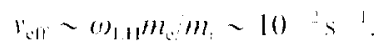

Let us estimate the characteristic time of encrgy

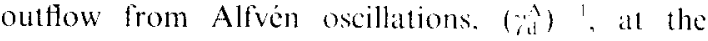
expense of LHO generation. A qualitative estimate may be obtained from

$$
\because W^{\lambda}={ }_{i n} W^{1 !} \text {. }
$$

Using values $W^{1} B_{0}^{2}-10^{+}$. (1) and (3), we obtain $\therefore A-10^{\prime}(1)_{A}$, where ()$_{A}$ is the Alfvén wave frequency. The linear growth rate of Alfven wave generation by anisotropic protons of the ring current during the eyclotron interaction has the form (Kennel and Petschek. 1966):

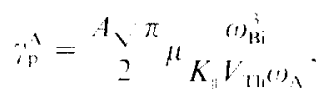

where $A$ is the temperature anisotropy of the ring current protons and $\mu$ is the relative concentration of anisotropic particles. For the relative concentration of hot particles $\mu-10$ and $\left.V_{\mathrm{A}} V_{\mathrm{Th}} \sim 10\right)^{1}\left(V_{\mathrm{Hh}}\right.$ is the thermal speed of hot protons) we have $\beta_{i} \sim 10$ I ("). Comparing $y_{\text {in }}$ and we may assume that the considered process may serve as one of the mechanisms restricting the level of the Allven turbulence in the region of its generation, i.e. in the ring current region.

Let us note, in conclusion, that the present results are of a qualitative nature. To clarify the authenticity of the suggested physical mechanisms, a detailed quantitative description is required. as well as the comparison of theoretical results with experimental da1a.

\section{REFERENCES}

Akhiezer. A. 1. (Editor) (1974) Plasma Electrodinamicy. Natukia, Moscow.

Galeev, A. А. (1975) Plasma turbulence in magnetosphere with special regard to plasma heating. in Physics of the Hot Plasna in the Magnetosphere. p. 251. Plenum Press, New York. 
Kennel, C. F. and Petschek, H. E. (1966) Limit on stably trapped particle fluxes. J. geophys. Res. 71, 1.

Korth, A., Kremser, G., Perraut, S. and Roux, A. (1984) Interaction of particles with ion cyclotron waves and magnetosonic waves. Observations from GEOS I and GEOS 2. Planet. Space Sci. 32, 1393.

Lyons, L. R. and Williams, D. J. (1984) Quantitative Aspects of Magnetospheric Physics. D. Reidel, Dordrecht.

Makarenko, V. N. and Tupchenko, A. M. (1988) Saturation of the level of Alfven fluctuations in the magnetospheric plasma. Geophys. J. 10,68.

Musher, S. L., Rubenchik, A. M. and Sturman, B. I. (1978) Plasma Phys. 20, 1131.

Musher, S. L., Rubenchik, A. M. and Shapiro, V. D. (1986) Nonlinear effects during the propagation of the ion beam across the magnetic field. ZhETF $\mathbf{9 0 , 8 9 0 .}$

Musher, S. L. and Sturman, B. I. (1975) On the collapse of plasma waves near the lower hybrid resonance. ZhETF Pis'ma Red. 22, 537.

Olsen, R. C., Shawhan, S. D., Gallagher, D. L., Green, J. L., Chappel, C. R. and Anderson, R. R. (1987) Plasma observations at the Earth's magnetic equator. J. geophys. Res. 92, 2385.

Sturman, B. I. (1976) The plasma turbulence near the lower hybrid resonance. ZhETF 71, 613.

Taylor, W. L., Parady, K. and Cahill, L. J. (1975) Explorer 45 observations of 1 to $30 \mathrm{~Hz}$ magnetic fields near the plasmapause during magnetic storms. J. geophys. Res. 80, 1271.

Young, D. T., Perraut, S., Roux, C., de Villedary, C., Gendrin, R., Korth, A., Kremser, G. and Jones, D. (1981) Wave particle interactions near $\Omega_{\mathrm{He}^{+}}$observed on GEOS1 and -2 . 1. Propagation of ion cyclotron waves in $\mathrm{He}^{+}$ rich plasma. J. geophys. Res. 85, 6755 . 